\title{
Simulating the time-dependent $d_{x^{2}-y^{2}}$ Ginzburg-Landau equations using the finite-element method
}

\author{
Qianghua Wang \\ Department of Physics, University of Hong Kong, Pokfulam, Hong Kong \\ and Department of Physics, Nanjing University, Nanjing 210093, China \\ Z. D. Wang \\ Department of Physics, University of Hong Kong, Pokfulam, Hong Kong
}

(Received 30 August 1996)

\begin{abstract}
The time-dependent Ginzburg-Landau equations describing a $d$-wave superconductor are simulated by the finite-element method. The equilibrium vortex structure in bulk samples, the nature of vortices in bulk and finite-size samples subject to various types of pinnings, and the transport behaviors are addressed. The extended finite-element method proves to be flexible to deal with various types of boundary conditions, desirable to simulate relaxation processes with very long time scales as well as the dynamics of vortices, especially in high- $\kappa$ superconductors. [S0163-1829(96)51246-4]
\end{abstract}

The symmetry of the order parameter in high-temperature superconductors and its effects on the transport properties are of current interest. Recently, a sign change of the order parameter on the Fermi surface, consistent with $d_{x^{2}-y^{2}}$ symmetry, has been reported. ${ }^{1-3}$ Thus it is interesting and necessary to consider what would come about from such a symmetry regarding the equilibrium and dynamic properties of the superconductor. Recently, the Ginzburg-Landau (GL) equations of a $d$-wave superconductor have been derived, ${ }^{4}$ on the basis of the microscopic Gor'kov equations. ${ }^{5}$ The interaction between electrons was assumed to be attractive in the $d$-wave channel and repulsive in the $s$-wave channel, so that the uniform superconductor always enjoys a pure $d$-wave pairing state. The GL free-energy functional for a twodimensional $d$-wave superconductor can be expressed in terms of two order parameters, $S(\mathbf{r})$ and $D(\mathbf{r}):^{4}$

$$
\begin{aligned}
G= & \int d \Omega 2 \alpha_{s}|S|^{2}-\alpha_{d}|D|^{2}+\frac{4}{3}|S|^{4}+\frac{1}{2}|D|^{4}+\frac{8}{3}|S|^{2}|D|^{2} \\
& +\frac{2}{3}\left(S^{* 2} D^{2}+\text { H.c. }\right)+2|\boldsymbol{\Pi} S|^{2}+|\boldsymbol{\Pi} D|^{2}+\left(\Pi_{x} S \Pi_{x}^{*} D^{*}\right. \\
& \left.-\Pi_{y} S \Pi_{y}^{*} D^{*}+\text { H.c. }\right)+\left(\boldsymbol{\nabla} \times \mathbf{A}-\mathbf{H}_{e}\right)^{2} .
\end{aligned}
$$

Here $\boldsymbol{\Pi}=i \boldsymbol{\nabla} / \kappa+\mathbf{A}, \quad \kappa$ being the GL parameter, $\alpha_{s}$ $=\alpha_{s 0} /\left(1-T / T_{c}\right) \quad$ and $\quad \alpha_{d}=\ln \left(T_{c} / T\right)$, where $\alpha_{s 0}$ $=4\left(1+2 V_{s} / V_{d}\right) / N(0) V_{d}$ is a positive constant, with $V_{s}>0\left(-V_{d}<0\right)$ being the effective interaction strength in the $s(d)$ channel, and $N(0)$ the density of states at the Fermi level, and finally $H_{e}$ is the applied magnetic field. Equation (1) is understood to be dimensionless according to the following normalization: the order parameters are normalized by $\Delta_{0}=\sqrt{4 / 3 \alpha}$ where $\alpha=7 \zeta(3) / 8\left(\pi T_{c}\right)^{2}$, the space by the magnetic penetration depth $\lambda$, and the vector potential by $\Phi_{0} / 2 \pi \xi$ and with $\Phi_{0}=h / 2 e$ being the flux quantum and $\xi$ the coherence length. The time-dependent GL equations, extended from the stationary equations ensuing from Eq. (1), may be written as ${ }^{6}$

$$
\begin{gathered}
{\left[\eta_{s} \partial_{t}+\alpha_{s}+\frac{4}{3}\left(|S|^{2}+|D|^{2}\right)+\mathbf{\Pi}^{2}\right] S+\frac{2}{3} D^{2} S^{*}} \\
\quad+\frac{1}{2}\left(\mathbf{\Pi}_{x}^{2}-\boldsymbol{\Pi}_{y}^{2}\right) D=0, \\
{\left[\eta_{d} \partial_{t}-\alpha_{d}+\frac{8}{3}|S|^{2}+|D|^{2}+\boldsymbol{\Pi}^{2}\right] D+\frac{4}{3} S^{2} D^{*}} \\
\quad+\left(\mathbf{\Pi}_{x}^{2}-\boldsymbol{\Pi}_{y}^{2}\right) S=0,
\end{gathered}
$$

$$
\begin{aligned}
\frac{\partial \mathbf{A}}{\partial t}+ & \boldsymbol{\nabla} \times \boldsymbol{\nabla} \times A+\left\{S^{*} \boldsymbol{\Pi} S+\frac{1}{2} D^{*} \boldsymbol{\Pi} D+\frac{1}{2}\left[S^{*}\left(\boldsymbol{\Pi}_{x}-\boldsymbol{\Pi}_{y}\right) D\right.\right. \\
& \left.\left.+D^{*}\left(\boldsymbol{\Pi}_{x}-\boldsymbol{\Pi}_{y}\right) S\right]+ \text { H.c. }\right\}-\boldsymbol{\nabla} \times \mathbf{H}_{e}=0,
\end{aligned}
$$

where $\Pi_{k}=\hat{x}_{k} \Pi_{k}, \eta_{s}$ and $\eta_{d}$ are two phenomenological constants characterizing the relaxation rate of the $s$ - and $d$-wave order parameters, respectively, and the time $t$ is normalized by $\sigma_{n} \lambda^{2}$ with $\sigma_{n}$ being the normal-state conductivity of the superconductor. We shall take simply $\eta_{s}=2 \eta_{d}=1$ (see, e.g., Ref. 6). In the above equations, we have assumed such a gauge that the electro-static potential does not appear.

The notion of the fourfold symmetry in the quasiparticle spectrum in the vortex state of a $d$-wave superconductor was elucidated theoretically by Maki and co-workers, ${ }^{7}$ who provide a nice and simple interpretation for the experimental results of Keimer et al. and Maggio-Aprile et al. ${ }^{8}$ that an unconventional oblique or even square vortex lattice may be the most stable configuration at low temperatures. Indeed, the latter phenomenon was also reproduced in earlier simulations $^{9}$ (based on the numerical-relaxation method ${ }^{10}$ ) of the vortex lattice in a $d$-wave superconductor, and was attributed to the coupling to the fourfold symmetric $s$-wave component of the order parameter induced by the gradient of the $d$-wave component (see also Ref. 11). However, the GL parameter $\kappa$ was taken to be $\kappa=2,{ }^{9}$ which is a bit too small to represent a realistic high- $T_{c}$ superconductor. Simulations of the mixed-state high- $\kappa$ superconductors might become difficult in the context of usual discretization schemes due to the large ratio between the two relevant length scales $\xi$ and 
$\lambda$ and the highly conditional convergence of the iterations. On the other hand, although the relaxation method ${ }^{10}$ the authors used in their simulations is well established for the purposes of calculating the equilibrium properties, it has to be extended to simulate the vortex dynamics. Furthermore, vortices in finite-size $d$-wave superconductors have not yet been examined by the GL equations. Motivated by these considerations, we resort to the time-dependent GL equations and simulate them by a derivative of an earlier method based on the finite-element method. ${ }^{12}$

The basic idea in the finite-element method (see, e.g., Ref. 13) is to expand, in each element cell of the sample, the functions to be solved by a complete set of piecewise shape functions (being unitary at the specified nodal points on the boundary of the cell), and to enforce orthogonality between any of the shape functions and the residual of the governing equations. The latter condition, combined with the continuity condition across the element cells and the boundary conditions concerned, determines the expanding coefficients and thus the approximate solution, and ensures that the free energy is stationary, in the static case, against any small variations that could also be presumably expanded by the shape functions. The completeness of the shape functions guarantees that the solution converges to the exact solution with decreasing volume of the element. It is clear that in the static situations, the finite element method, starting from minimizing the free energy in each element cell, serves as a global minimizer of the total free energy by assembling the elements. In the time-dependent situation, the functions are expanded as the same shape functions but with time-dependent coefficients, which are to be solved by the usual Euler schemes. The details of the finite-element method extended to our case are presented elsewhere. ${ }^{14}$

For comparison, we first present in Fig. 1 the static single vortex profile in an $8 \lambda \times 8 \lambda$ unit cell with periodic boundary condition (see Refs. 9 and 10 for details). Here $\kappa=2$ and $\alpha_{s}=-\alpha_{d}=1$. We have used biquadratic polynomial shape functions and triangular element cells, each of size $0.5 \xi \times 0.5 \xi / 2$. The explicit Euler scheme is employed to save the storage memory (since the effective coefficient matrix can be diagonalized once and forever by the so-called lumping technique, see, e.g., Ref. 13). As an expense of this scheme, we have to use a time step lower than $10^{-3}$. The initial condition is a pure $d$-wave state. The simulation converges smoothly and steadily. The fourfold symmetry as seen from the plot of the $s$-wave amplitude in Fig. 1(a) is evident. It is also discernible far from the vortex core in Fig. 1(b), where the amplitude of the $d$-wave component is plotted. All of these results are comparable with the counterparts in Ref. 9 , where more details on the single vortex can be found (see also Ref. 15).

Figure 2 shows the vortex lattice at $\kappa=10$ and $\alpha_{s}=-\alpha_{d}=1$, replicated from the simulation results in a rectangular unit cell (with an area of $18.862 \xi^{2}$ ) with two vortices. The ratio of the side lengths of the rectangular is $r=\sqrt{3}$, which corresponds to a triangular lattice. We have not plotted the field distribution since it is essentially uniform over the cell at this stage of vortex density. Using the implicit Euler method, the iteration time step can be varied fairly arbitrarily up to $\Delta t=100$, and the iteration converges after only tens to a few hundreds of iteration steps with $\Delta t$

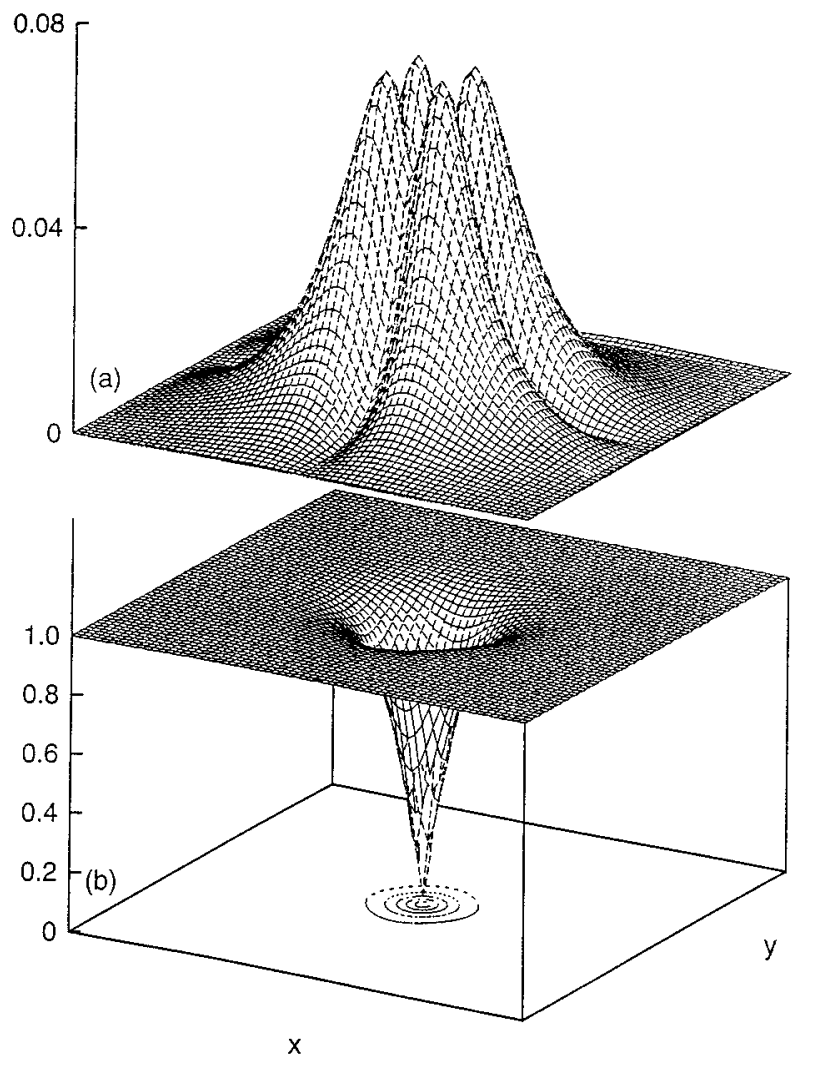

FIG. 1. Surface-contour plots of a single vortex. (a) The $s$-wave amplitude. (b) The $d$-wave amplitude.

being of order 10 . Therefore, we can safely reach the steadystate solution of the GL equation and thus give reliable results regarding the equilibrium properties. With fixed unit cell area as above, we have also obtained steady-state solutions of other structures (or other values of $r$ ) at various $s$-wave channel parameters $\alpha_{s}$ (not plotted here). In a realistic sample, the equilibrium vortex structure corresponds to the optimal value of $r$ that minimizes the free energy. Table I presents the free energy density $\mathcal{G}$ versus $r$ and $\alpha_{s}$ [the applied field $H_{e}$ disappears from Eq. (1) since it is the Helmholtz free energy that is under consideration]. In principle, $\alpha_{d}$ and $\alpha_{s}$ are temperature dependent from definitions. However we can fix $\alpha_{d}=1$ by rescaling the order parameters, the
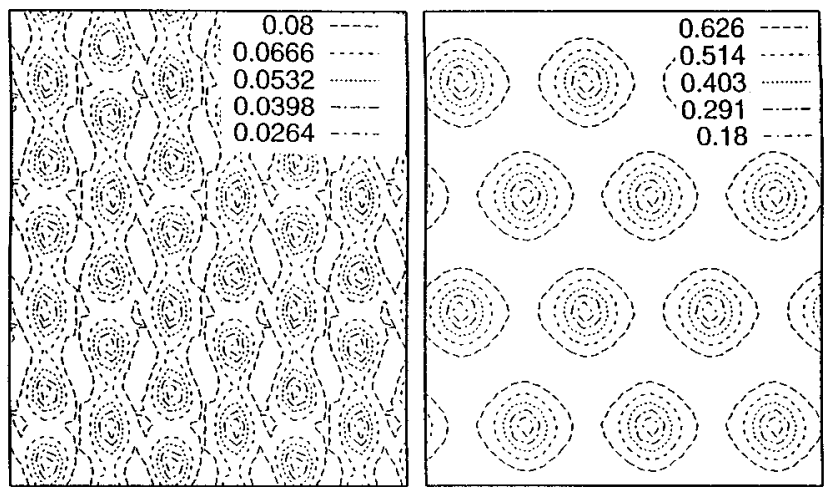

FIG. 2. Contour plots of a triangular vortex lattice. The left (right) panel is for the $s$ - $(d-)$ wave amplitude. 
TABLE I. Free energy density as a function of $r$ and $\alpha_{s}$. See the text for other parameters.

\begin{tabular}{|c|c|c|c|c|c|c|c|c|}
\hline$\alpha_{s}$ & 0 & & 1 & & 2 & & $\infty$ & \\
\hline$r$ & $\sqrt{3}$ & 1.35 & $\sqrt{3}$ & 1.35 & $\sqrt{3}$ & 1.35 & $\sqrt{3}$ & 1.35 \\
\hline $\mathcal{G}$ & 44.3312 & 44.3251 & 44.3255 & 44.3245 & 44.3296 & 44.3227 & 44.3381 & 44.3384 \\
\hline
\end{tabular}

space, and the vector potential. For $\alpha_{s}=0,1,2$, we reproduce the earlier result that an oblique lattice (with $r<\sqrt{3}$ ) is more stable than the conventional triangular lattice. ${ }^{9,11}$ This effect has been ascribed to the fourfold symmetry of the system: restoring of the fourfold symmetry lowers the potential energy while lifting the kinetic energy and the field energy. With increasing $\alpha_{s}$ (or increasing temperature below and near $T_{c}$ ), the $s$-wave component is suppressed and plays less a role. Near $T_{c}$, the system is well described by the single order parameter $D$, being equivalent to a conventional isotropic system. Indeed, at $\alpha_{s}=\infty$ (or in the absence of the $s$-wave component), we recover the classical result that the triangular lattice with $r=\sqrt{3}$ is the most stable. Concluding the above results we see that as the temperature (or $\alpha_{s}$ ) increases from below $T_{c}$, the vortex lattice would undergo a crossover from the square to the triangular structure. Our results for various values of $\kappa \geqslant 2$ give a similar picture, and are therefore complementary to the earlier conjecture. Such a picture seems to be consistent with the experimental observations $^{8}$.

Figure 3 is the evolution of the vortices in a $\kappa=3$ tapelike sample under an applied current $J_{y}=0.1$ along the longitudinal (or $y$ ) direction of the tape and an applied field $H_{e}=1$ normal to the tape. We use periodic boundary conditions in $y$ and open boundary condition in the transverse $(x)$ direction. In obtaining Figs. 3 , the original $2 \lambda \times 2 \lambda$ unit cell has been replicated twice in the $y$ (or vertical) direction. The vortices penetrate from the right boundary, move in the direction of the Lorentz force in the interior, and annihilate at the left boundary. The $s$-wave component of the order parameter is seen to ride over the $d$-wave component adiabatically. It should be pointed out that the algorithm described in the previous section applies best for relaxation problems with no applied current. While in the presence of an applied current, the vector potential would increase endlessly in the fixed gauge (in the absence of an electrostatic potential), and one would soon lose accuracy in the iteration. The heuristic reason is as follows. A large amplitude of the vector potential amounts to a large gradient in the phase $\varphi$ (and thus in the real and imaginary parts) of the order parameter so as to keep the physically meaningful gauge invariant phase $\varphi-\kappa \int \mathbf{A} \cdot d \mathbf{r}$ varying reasonably slowly in space. This requires a smaller and smaller element cellular volume to approximate accurately violent spatial variations of the relevant functions when the average vector potential $\mathbf{A}_{0}=\langle\mathbf{A}\rangle$ becomes large. To get rid of this problem, we have performed global gauge transformations after each step of iteration,

$$
\mathbf{A} \rightarrow \mathbf{A}-\mathbf{A}_{0}, \quad \varphi \rightarrow \varphi-\kappa \int \mathbf{A}_{0} \cdot d \mathbf{r},
$$

which is justified for adiabatic dynamics, e.g., when the applied current is significantly lower than the depairing current so that the vortices can move steadily. In this case, the in- stant spatial average of the electric field is simply given by $\mathbf{E}=\mathbf{A}_{0} / \Delta t$. Therefore, the transport behavior of the system can be simulated easily. The above modification for the dynamic case applies equally well in open boundary problems and periodic boundary problems. We have monitored the time dependence of $E=A_{0, y} / \Delta t$ (the lowest panel) to find that on a steady background there are quasiperiodic peaks in $t$, which signal the sudden events when the vortices enter or leave the sample. The second-order response, namely, the Hall effect, could also be implemented in the simulations easily. Although the $s$-wave amplitude is nonzero here, we have found no essential difference between the longitudinal transport behaviors of the vortices in the present case and in the case where the superconductor is described by the conventional single-component $s$-wave GL equations. This is a desired result as confirmed exhaustively by experiments. However, we expect that the detailed field and temperature dependence of the longitudinal resistivity as well as the Hall resistivity would be affected by the presence of the ambient $s$-wave component. ${ }^{14}$

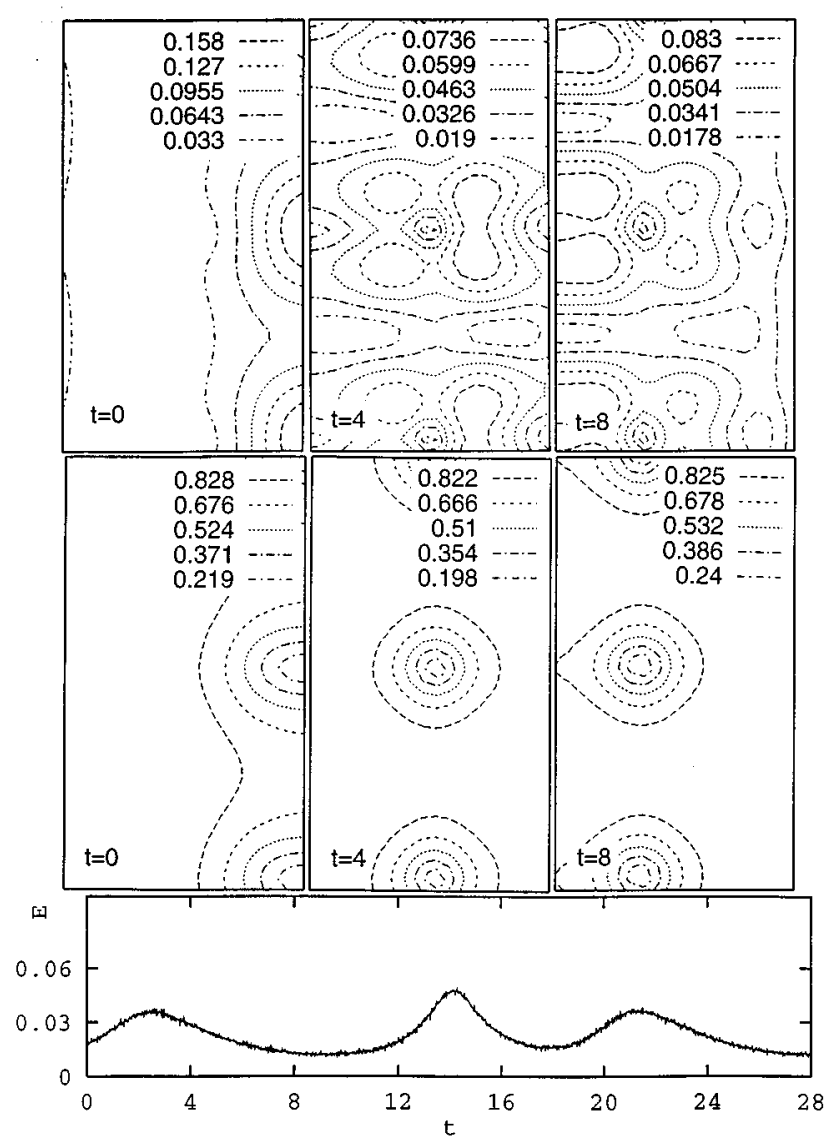

FIG. 3. Evolution of vortices in a tapelike sample. The upper (middle) panels are contour plots of the $s-(d$-) wave amplitude. The lowest panel is the time dependence of the electric field. 


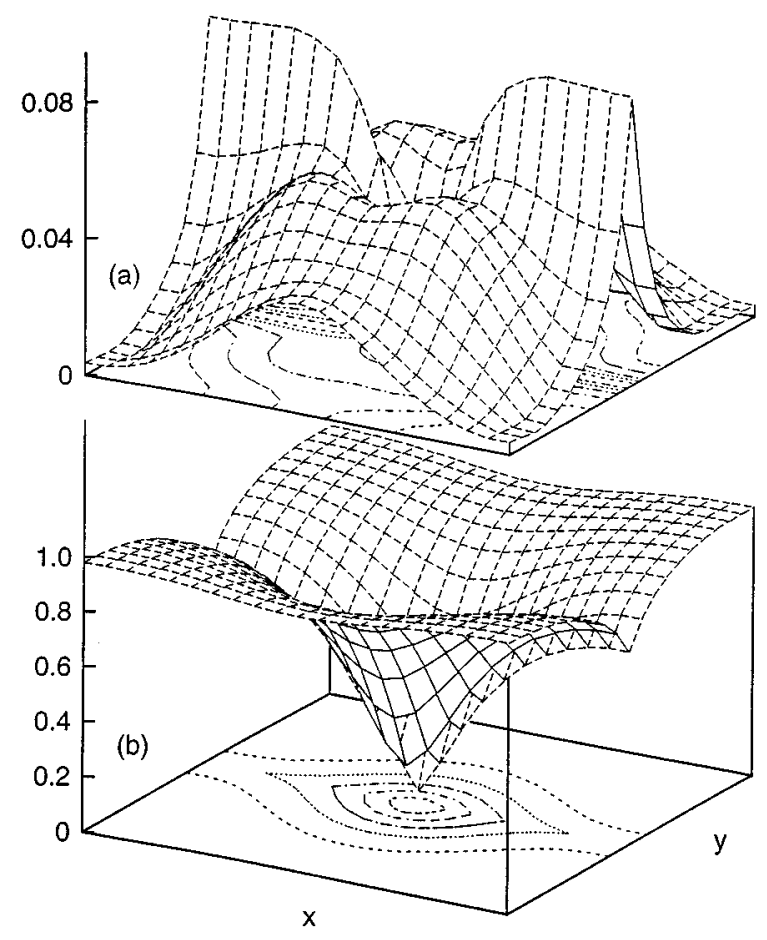

FIG. 4. Surface-contour plots of a vortex in a unit cell with a twin boundary. (a) The $s$-wave amplitude. (b) The $d$-wave amplitude

Next, we examine the effects of a twin boundary on the vortices. To be clear of the sample boundary, we use again the periodic boundary condition with one flux quantum in a $1.9 \lambda \times 1.9 \lambda$ unit cell. Furthermore, a periodic array of twin boundaries (with a transverse spacing of $L=1.9 \lambda$ ) are assumed, $\alpha_{i}=\alpha_{i, 0}+V_{i} \Sigma_{k} \delta(y-k L)$, where the subscript $i$ stands for $s$ or $d$. Here we assume that the twin boundaries are created by some chemical method in a parent crystal with no misorientations. Otherwise, two twin boundaries are needed in one unit cell to apply the periodic boundary condition. In Fig. $4, \alpha_{s, 0}=-\alpha_{d, 0}=1$ and $V_{s}=V_{d}=0.1$. In contrast to the usual discretization scheme, such twin boundaries can be easily implemented in the finite element method as if they were extra boundaries of the samples with the following discontinuity:

$$
\begin{gathered}
\frac{i}{\kappa} \hat{n} \cdot\left[\Pi \mathbf{\Pi} S+\frac{1}{2}\left(\boldsymbol{\Pi}_{x}-\boldsymbol{\Pi}_{y}\right) D\right]_{-}^{+}=-V_{s} S, \\
\frac{i}{\kappa} \hat{n} \cdot\left[\boldsymbol{\Pi} D+\left(\boldsymbol{\Pi}_{x}-\boldsymbol{\Pi}_{y}\right) S\right]_{-}^{+}=-V_{d} D,
\end{gathered}
$$

which follows immediately from an integration of Eqs. (2) and (3) along the normal direction $\hat{n}$ of the twin boundary over an infinitesimal interval. Here []$_{-}^{+}$means the jump of the argument across the boundary. These equations are substituted as natural boundary conditions in the finite-element scheme. ${ }^{13,14}$ Figures 4(a) and 4(b) are the surface-contour plots of the $s$-wave and $d$-wave amplitudes, respectively. Along the twin boundary, the $d$-wave order parameter is suppressed while the $s$-wave order parameter is greatly enhanced.

Due to limited space, not presented here are the simulation results for superconductors with inhomogeneities arising from finite variation of the physical properties (such as $\alpha_{s, d}$ and $\lambda),{ }^{16}$ which can also be easily implemented in the simulation scheme. A comparative study on the equilibrium and transport behaviors of vortices in the context of conventional and unconventional GL theories is being undertaken.

We are grateful to Professor Qiang Du for helpful discussions. This work was supported by the RGC grant of Hong Kong, and the Natural Science Foundation of China.
${ }^{1}$ D. A. Wollman, D. J. Van Harlingen, W. C. Lee, D. M. Ginsberg, and A. J. Leggett, Phys. Rev. Lett. 71, 2134 (1993).

${ }^{2}$ C. C. Tsuei et al., Phys. Rev. Lett. 73, 593 (1994).

${ }^{3}$ J. H. Miller, Jr. et al., Phys. Rev. Lett. 74, 2374 (1995).

${ }^{4}$ Y. Ren, J. H. Xu, and C. S. Ting, Phys. Rev. Lett. 74, 3680 (1995); Phys. Rev. B 53, 2249 (1996); J. H. Xu, Y. Ren, and C. S. Ting, ibid. 52, 7663 (1995).

${ }^{5}$ L. P. Gor'kov, Zh. Éksp. Teor. Fiz. 36, 1918 (1959) [Sov. Phys. JETP 9, 1364 (1960)].

${ }^{6}$ Y. Ren, J. H. Xu, and T. S. Ting, J. Phys. Chem. Solids 56, 1749 (1995).

${ }^{7}$ H. Won and K. Maki, Europhys. Lett. 30, 421 (1995); N. Schopohl and K. Maki, Phys. Rev. B 52, 490 (1995); H. Won and K. Maki, ibid. 53, 5927 (1996).

${ }^{8}$ B. Keimer et al., J. Appl. Phys. 76, 6788 (1994); B. Keimer et al., Phys. Rev. Lett. 73, 3459 (1994); I. Maggio-Aprile, C. Renner, A. Erb, E. Walker, and Ø. Fisher, ibid. 75, 2754 (1995).
${ }^{9}$ J. H. Xu, Y. Ren, and C. S. Ting, Phys. Rev. B 53, R2991 (1996).

${ }^{10}$ Z. D. Wang and C. R. Hu, Phys. Rev. B 44, 918 (1991).

${ }^{11}$ A. J. Berlinsky, A. L. Fetter, M. Franz, C. Kallin, and P. I. Soininen, Phys. Rev. Lett. 75, 2200 (1995); M. Franz, C. Kallin, P. I. Soininen, A. J. Berlinsky, and A. L. Fetter, Phys. Rev. B 53, 5795 (1996); A. J. Berlinsky, A. L. Fetter, M. Franz, C. Kallin, and P. I. Soininen, J. Phys. Chem. Solids 56, 1695 (1995); 56, 1619 (1995).

${ }^{12}$ Q. Du, M. D. Gunzburger, and J. S. Peterson, Phys. Rev. B 51, 16194 (1995).

${ }^{13}$ D. S. Burnett, Finite Element Analysis: From Concepts to Applications (Addison-Wesley, Reading, MA, 1987).

${ }^{14}$ Q. H. Wang and Z. D. Wang (unpublished).

${ }^{15}$ G. E. Volovik, Pis'ma Zh. Éksp. Teor. Fiz. 58, 457(1993) [JETP Lett. 58, 469 (1993)].

${ }^{16}$ W. Xu, Y. Ren, and C. S. Ting, Phys. Rev. B 53, 12481 (1996). 